\title{
Performance Evaluation of DCT and DWT Features for Blind Image Steganalysis using Neural Networks
}

\author{
Manisha Saini \\ Student, M.Tech. (CSE) \\ Department of CSE/IT \\ ITM University, Gurgaon, Haryana, India
}

\author{
Rita Chhikara \\ Assistant Professor \\ Department of CSE/IT \\ ITM University, Gurgaon, Haryana, India
}

\begin{abstract}
In this paper, we are comparing 72 DWT feature set with 274 DCT feature set on the basis of parameters MSE (Mean square error), Accuracy and time for Blind Image Steganalysis. Dataset is created consisting of cover images and stego images, which are obtained by using two steganography tools steghide and outguess.Extracted features are then fed to Neural network Back propagation classifier, to compare the performance.Experimental results show that DCT feature set has higher performance as compared to DWT feature set.
\end{abstract}

\section{Keywords}

DCT, DWT, Neural network, Outguess, Steganography, Steganalysis, StegHide.

\section{INTRODUCTION}

Steganography[1] is a technique of hiding the message in carrier medium like audio, video, text, image etc with the help of some steganography tools. There is wide variety of steganography tools available like Stool, StegHide, F5, JPHide etc. Counter part of steganography is steganlysis, it is used to detect the message hidden in the carrier medium. There are two kinds of steganalysis method [2]. (i) Target steganlysis, where particular kind of steganography algorithm which is used to hide the message is known. (ii)Blind/Universal Steganlysis, where the particular kind of steganography tool used to hide the message is not known. Wide availability of steganography tools for hiding information led to an increased interest in steganalysis. Evidence has been found that terrorists have used steganography technique to hide some information. Hence Steganlysis plays an important role in digital forensic to help overcome such threats. Steganography hides data in Spatial Domain and Transform Domain. Main difference between spatial and transform domain is that in spatial domain data embedding in pixels is done directly where as in transform domain images are first converted to DCT (Discrete cosine transform ), DFT (Discrete Fourier transformation) or DWT (Discrete Fourier transform ) domain and then the message is embedded inside the image. So advantage of this transform over spatial method is that it is more secure against statistical attacks.

In DCT,[3] first step is to divide an image into blocks of size 8 X8 \& then DCT transform is applied, using JPEG standard quantization table, then we get quantized coefficients. There are two types of quantized coefficients (1) DC coefficients, are low frequency components contains maximum information (2) AC Coefficients, are high frequency component contain least information.

DCT equation is shown below [4] -

$$
C(u, v)=\alpha(u) \alpha(v) \sum_{x=0}^{N-1} \sum_{y=0}^{N-1} f(x, y) \cos \left[\frac{\pi(2 x+1) u}{2 N}\right] \cos \left[\frac{\pi(2 y+1) v}{2 N}\right] .
$$

In DWT ,[5][18] After applying Discrete wavelet transform on image we get 4 different subands (1)LL (2)HL (3)LH (4) $\mathrm{HH}$, where LL suband is low frequency component contain maximum information and other three subands (HL,LH,HH) are high frequency component contain less information. Main objective of DWT is space localization, can predict at which space we have low frequency component and at which space we have high frequency component. In DWT [6] there is no overlapping of 2D blocks and avoid block artificats as there in DCT.

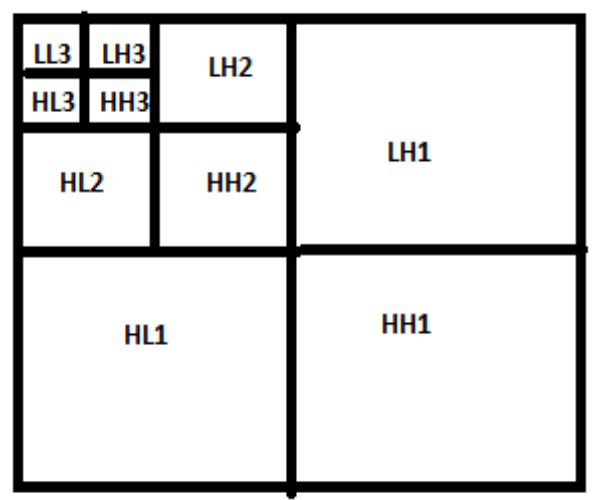

Fig 1: Three-level wavelet decomposition

The Rest of this paper is structured as shown Section 2 discusses the steganography tool used Section 3 discusses about DCT and DWT feature Section 4 neural network is used as the classifier. Experimental Result and Analysis is presented in Section 5. Conclusion is shown in Section 6.

\section{STEGANOGRAPHY ALGORITHMS}

\subsection{Outguess}

Outguess [7] is a universal steganographic tool that allows the addition of hidden information into the redundant bits of data sources.Outguess, was designed by Niels provos. Outguess is an advanced variant of Jsteg in that it uses PRNG-based scattering to complicate steganalysis. The embedding and extraction functions therefore each require a seed as additional parameter to initialize the PRNG. Choice of good random seed is crucial in field of computer security. Outguess algorithm is shown below [8]

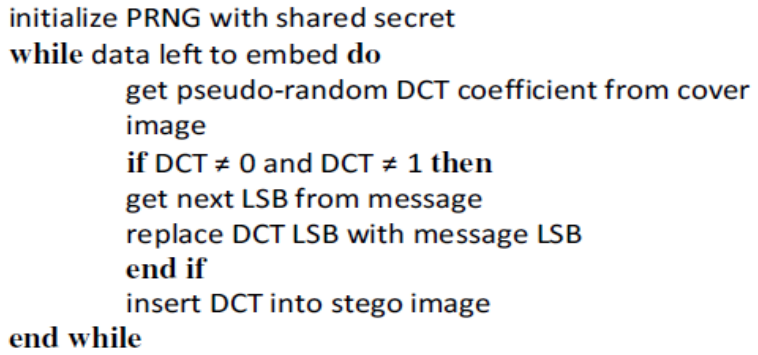




\subsection{StegHide}

Steghide is another steganography tool that is used to hide data in various kind of image \& audio-file [9].Steghide uses a graph - theoretic approach to Seganography[10].Hetzl and Mutzel designed a graph-theoretic approach for information hiding based on the idea of exchanging rather than overwriting pixels [8].Steghide is command line tool available for Unix platforms [9].

\section{Steghide embed -cf new.jpg -ef secret.jpg}

This command is used in ubuntu to embed image secret.jpg into cover image new .jpg

\section{Steghide extract - sf new.jpg}

Command is used to extract secrete.jpg image from cover image new.jpg with the help of secrete key.

\section{DCT AND DWT FEATURE}

\subsection{DCT Feature (penvy and fridrich 274)}

Jessica Fridrich and Tomas Pevny proposed [11] merged ' 193 extended DCT features 'with ' 81 averaged calibrated Markov features', so resulted feature set is $193+81=274$ which is extracted from DCT (Discrete Cosine Transform) domain is called new Merged feature set. Feature extracted are Global histogram, AC histograms, Dual histograms, Variation, Blockiness ,Co-occurrence matrix and Markov features.

\subsection{DWT Feature (farid 72)}

Farid proposed 72 feature set [12], [13], 4 features that are extracted from subands are mean, variance, skewness and kurtosis Formula for these 4 features is shown below[15]:-

$$
\begin{aligned}
E(x) & =\frac{1}{n} \sum_{k=1}^{n} x_{k} \\
\operatorname{Var}(x) & =\frac{1}{n-1} \sum_{i=1}^{n}\left(x_{i}-E(x)\right)^{2} \\
S(x) & =E\left[\left(\frac{x-E(x)}{\sqrt{(} \operatorname{Var}(x))}\right)^{3}\right] \\
K(x) & =E\left[\left(\frac{x-E(x)}{\sqrt{(\operatorname{Var}(x))}}\right)^{4}\right]
\end{aligned}
$$

Fig 2: Mean, Variance, skewness and kurtosis formula

The image decomposition used in farid paper, is based on separable quadrature mirror filters (QMFs).Decomposition contain vertical, horizontal and diagonal subands.

\section{NEURAL NETWORK CLASSIFIER}

The design of classifier[14][15] is another key element in Steganalysis.As good the classifier is better will be the classification rate and chances of getting error or misclassification would be less. Classifier is used to detect whether the image is stego image or cover image.

We have used neural network Tool, where input data given is feature extracted from images and since we have two classes, so target output is in binary form either 1 or 0 . Neural network tool will help us to select data, create and train a network, and evaluate its performance using mean square error, accuracy and percentage error. Some of the key features of neural network are (1) Non liner classifier (2) Learning is fast (3) High tolerances to noisy Data. Neural network classifier has three layers (1) Input layer (2) Output layer (3) Hidden layer. In our work, we have changed the number of hidden layer to see changes in output. (Mean square error (MSE) and accuracy are calculated in each case along with time taken).

Formula for Mean square error [17]is:-

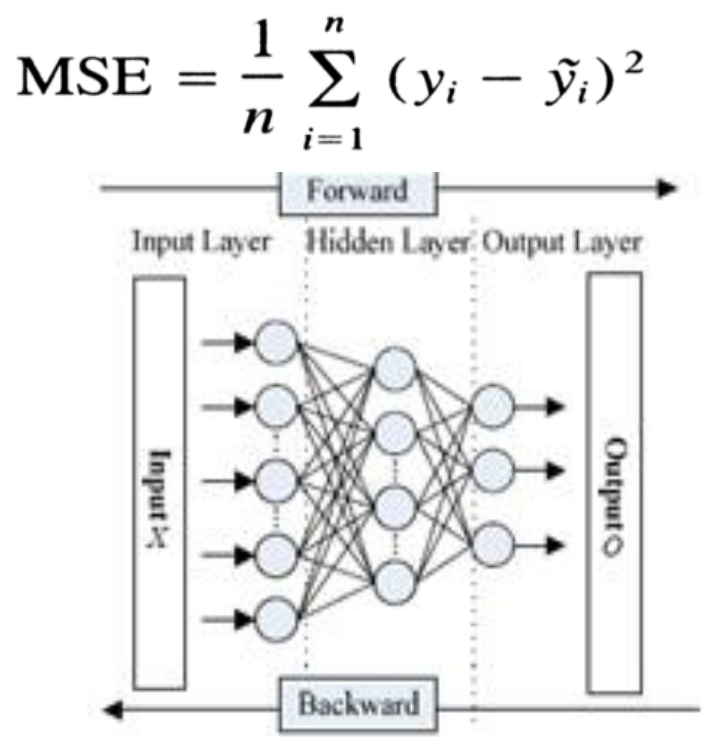

Fig 3: BP Neural Network

\section{EXPERIMENT RESULT AND ANALYSIS}

We have Cover Image Dataset consisting of 2,000 images downloaded from various sites and photographs of various sizes which are resized into 640X480 size. After applying two Steganography tools (1) StegHide (2) Outgues we get two set of 2,000 stego image. Various embedding capacities have been used to generate the dataset as shown in Table 1. So total 4,000 Images (cover plus stego) in one case, out of these 4000 images 2,800 is used for Training, 600 is used for Validation and 600 for Testing.

Table 1 . Various embedding capacities

\begin{tabular}{|l|l|}
\hline Outguess & StegHide \\
\hline $16 \times 16$ & $16 \times 16$ \\
\hline $32 \times 32$ & $64 \times 64$ \\
\hline $48 \times 48$ & $10 \times 10$ \\
\hline
\end{tabular}

As there are three kinds [16] of samples (1) Training samples are presented to the network during training, and the network is accustomed according to its error (2) Validation samples are used to evaluate network generalization, and to halt training when generalization stops improving.(3)Testing samples have no effect on training and so provide an independent measure of network performance during and after training After dividing the samples into training, validation and testing samples ,then features are extracted and extracted features from both cover and stego images are used to train the Neural network back propagation classifier.

Mean Squared Error and accuracy is calculated and time is noted down, where Mean Squared Error is the average squared difference between outputs and targets and Percent Error indicates the fraction of samples which are misclassified, Value of 0 means no misclassifications, 100 indicate maximum misclassifications. 
We try to retrain the network to see if there will be some changes, so we return to the panel and change the number of Hidden neurons i.e. 20 neurons to 30 neurons, in both case of DCT and DWT features and calculated mean square error (MSE) and accuracy again using MATLAB.

The Comparisons between DCT (274 feature) and DWT (72 feature) on the basis of parameters MSE, Accuracy and time, in both the case of stegonography tool Outguess and StegHide are listed in Table 2. When we increase the no of hidden neurons from 20 to 30 , Table 3. again shown the comparison difference between DCT and DWT.Both DCT and DWT take same time but accuracy is better for DCT in comparison to DWT.

\section{CONCLUSION}

We have created dataset of images consisting of cover and stego images, where stego images are obtained by applying two stegonagraphy tool StegHide and Outguess. Features are extracted from image from two transform domain DCT (274 features) and DWT (72 features). The features are fed to neural network back propagation classifier for detecting whether image is stego or cover.The performance of classifier is compared on the basis of parameter mean square error (MSE), accuracy and time taken. Experiment results have shown that i) DCT (274 feature) has higher accuracy as compared to DWT (72 feature) ii) even when we increase the no of hidden neurons still there is no significant change in the result.Future work shall be to expand the work for other steganography tools and spam feature set.

\section{REFERENCES}

[1] Abbas Cheddad, JoanCondell, Kevin Curran, PaulMcKevitt ,"Digital image steganography: Survey and analysis of current methods", Signal Processing90 (2010)727-752.

[2] Arooj Nissar,A.H. Mir,"Classification of steganalysis techniques: A study", Digital Signal Processing 20 (2010) 1758-1770.

[3] Seyed Mansour Hashemipour, Mohammad Rahmati,“A DCT Statistics-Based Universal Image Steganalysis",2012 Eighth International Conference on Intelligent Information Hiding and Multimedia Signal Processing.

[4] http://www.tutorialspoint.com/dip/Image_Transformatio ns.htm

[5] Shaker K. Ali , Zou Beijie, "Analysis and Classification of Remote Sensing by using Wavelet Transform and Neural Network", IEEE 2008 International Conference on Computer Science and Software Engineering.
[6] Archana Deshlahra,G.S.Shirnewar ,Dr. A.K. Sahoo,"A Comparative Study of DCT, DWT \& Hybrid (DCTDWT) Transform".

[7] Niels provos, www.outguess.org.

[8] Deepika Bansal, Rita Chhikara,"Performance Evaluation of Steganography Tools Using SVM and NPR Tool", 2014 Fourth International Conference on Advanced Computing \& Communication Technologies.

[9] http://steghide.sourceforge.net/

[10] S. Hetzl, P. Mutzel, A graph-theoretic approach tosteganography, in: Ninth IFIP TC-6 TC-11 International Conference, Lecture Notes in Computer Science, vol. 3677, 2005, pp. 119-12.

[11] Tom'a s Pevn'y, Jessica Fridrich, "Merging Markov and DCT Features for Multi-Class JPEG Steganalysis", The International Society for Optical Engineering, volume 6505, pp.28-40, 2007.

[12] Hany Farid, "Detecting Hidden Messages Using Higherorder Statistical Models", Proc. IEEE Symp. Int'l Conf. on Image Processing (ICIP 2000), IEEE Press, Sep.2002,pp.905-908,doi:10.1109/ICIP.2002 1040098.

[13] S. Lyu, and H. Farid, "Steganalysis Using Higher-order Image Statistics",Trans. Information Forensics and Security,vol.1,Jan.2006,pp.111-119,doi:10.1.1.116.1418.

[14] Yun Q. Shi, Guorong Xuan, Dekun Zo1, Jianjiong Gao,Chengyun Yang, Zhenping Zhang, Peiqi Chai, Wen Chen, Chunhua Chen, "Image Steganalysis Based on Moments of Characteristic Functions Using Wavelet Decomposition, Prediction-Error Image, and Neural Network",IEEE,2005.

[15] Yuan Liu, Li Huang, Ping Wang, Guodong Wang, "A Blind Image Steganalysis Based on Features from Three Domain"(2008 Chinese Control and Decision Conference (CCDC 2008)).

[16] Neural Network Pattern Recognition Tool

[17] Paul W. Mielke Jr, Kenneth J. Berry ,Christopher W. Landsea and William M. Gray, "Artificial Skill and Validation in Meteorological Forecasting", American Meteorological Society (AMS).

[18] Syed Ali Khayam,"The Discrete Cosine Transform (DCT): Theory and Application”. Department of

[19] Dai Zhonghua , Xiong Qi , Peng Yong and Gao Haihui,"Research on the Large Scale Image Steganalysis Technology Based on Cloud Computing and BP Neutral Networ-kTechnology", IEEE, 2012.

Table 2. Experimental Result with 20 Hidden neurons

\begin{tabular}{|c|c|c|c|c|c|c|c|c|c|c|c|c|}
\hline \multicolumn{13}{|c|}{20 neurons } \\
\hline & \multicolumn{6}{|c|}{ DCT (274 Features) } & \multicolumn{6}{|c|}{ DWT (72Features) } \\
\hline & \multicolumn{3}{|c|}{ Outguess } & \multicolumn{3}{|c|}{ StegHide } & \multicolumn{3}{|c|}{ Outguess } & \multicolumn{3}{|c|}{ StegHide } \\
\hline & Accuracy & Time & MSE & Accuracy & Time & MSE & Accuracy & Time & MSE & Accuracy & Time & MSE \\
\hline Training & 99.964 & 0.03 & $3.966 \mathrm{e}^{-4}$ & 98.929 & 0.04 & $1.1341 \mathrm{e}^{-2}$ & 66.69429 & 0.04 & $2.134 \mathrm{e}^{-1}$ & 54.4642 & 0.02 & $2.480 \mathrm{e}^{-1}$ \\
\hline Validation & 99.833 & 0.03 & $1.6831 \mathrm{e}^{-3}$ & 98.333 & 0.04 & $1.1671 \mathrm{e}^{-2}$ & 64.33333 & 0.04 & $2.174 \mathrm{e}^{-1}$ & 51.1667 & 0.02 & $2.508 \mathrm{e}^{-1}$ \\
\hline Testing & 99.667 & 0.03 & $3.0696 \mathrm{e}^{-3}$ & 97 & 0.04 & $2.6001 \mathrm{e}^{-2}$ & 67.33334 & 0.04 & $2.155 \mathrm{e}^{-1}$ & 45.8334 & 0.02 & $2.53 \mathrm{e}^{-1}$ \\
\hline
\end{tabular}


Table 3. Experimental Result with 30 Hidden neurons

\begin{tabular}{|c|c|c|c|c|c|c|c|c|c|c|c|c|}
\hline \multicolumn{13}{|c|}{30 neurons } \\
\hline & \multicolumn{6}{|c|}{ DCT (274 Features) } & \multicolumn{6}{|c|}{ DWT (72Features) } \\
\hline & \multicolumn{3}{|c|}{ Outguess } & \multicolumn{3}{|c|}{ StegHide } & \multicolumn{3}{|c|}{ Outguess } & \multicolumn{3}{|c|}{ StegHide } \\
\hline & Accuracy & Time & MSE & Accuracy & Time & MSE & Accuracy & Time & MSE & Accuracy & Time & MSE \\
\hline Training & 99.964 & 0.03 & ${ }_{4}^{5.3722 \mathrm{e}^{-}}$ & 98.179 & 0.02 & $\begin{array}{l}1.161 \\
\mathrm{e}^{-2}\end{array}$ & 65 & 0.04 & ${ }_{1}^{2.1596 \mathrm{e}^{-}}$ & 52.8929 & 0.02 & $\begin{array}{l}2.4909 \mathrm{e}^{-} \\
1\end{array}$ \\
\hline Validation & 100 & 0.03 & $2.8752 \mathrm{e}^{-}$ & 97.833 & 0.02 & ${ }_{2}^{2.119 \mathrm{e}^{-}}$ & 66.667 & 0.04 & ${ }_{1}^{2.2099 \mathrm{e}^{-}}$ & 54.5 & 0.02 & $\begin{array}{l}2.4802 \mathrm{e}^{-} \\
1\end{array}$ \\
\hline Testing & 99.6667 & 0.03 & $3.125 \mathrm{e}^{-}$ & 97.5 & 0.02 & ${ }_{2}^{1.792 \mathrm{e}^{-}}$ & 60 & 0.04 & ${ }_{1}^{2.2738 \mathrm{e}^{-}}$ & 50.8334 & 0.02 & $2.520 \mathrm{e}^{-}$ \\
\hline
\end{tabular}

